\title{
Controlled Growth of Aligned Polymer Nanowires
}

\author{
Hao Fang, ${ }^{\dagger, \dagger}$ Wenzhuo Wu, ${ }^{\dagger}$ Jinhui Song, ${ }^{\dagger}$ and Zhong Lin Wang $*, \dagger$ \\ School Materials Science and Engineering, Georgia Institute of Technology, Atlanta, Georgia 30332-0245, \\ Department of Advanced Materials and Nanotechnology, College of Engineering, Peking University, 100084 \\ Beijing, China
}

Received: July 24, 2009; Revised Manuscript Received: August 20, 2009

\begin{abstract}
We have developed a one-step method to fabricate large-scale polymer nanowire (PNW) arrays of any organic materials, with a good control over the density and length. The PNWs are formed by ion etching a polymer film that is covered with a thin layer of metal nanoparticles, which serves as the "mask" for creating the surface roughness required for creating the PNWs. Our study demonstrates an effective approach for creating functional organic NW arrays for applications in sensors, electronics, biomaterials, and energy materials.
\end{abstract}

Nanowires (NW) of semiconductor and functional oxides are the fundamental units for fabricating nanoscale devices for photonics, electronics, sensors, bioscience, and energy science. ${ }^{1-7}$ Growth of orientation-, dimensionality-, and location-controlled NWs is the key for controlling the performance of nanodevices toward nanomanufacturing. ${ }^{8-10} \mathrm{NW}$ arrays of inorganic materials are usually grown by physical approach via a vapor-liquid-solid $(\mathrm{VLS})^{11}$ or vapor-solid-solid (VSS) ${ }^{12,13}$ process at high temperature and a wet chemical approach using solvent-thermal or hydrothermal process. ${ }^{14-16}$ The alignment of inorganic NWs is usually achieved using either catalyst-guided growth together with the epitaxial relationship with the substrate or the template technique. ${ }^{17,18}$ Polymer-based NWs have potential applications in sensors, organic light-emitting diodes (OLEDs), field-effect transistors (FETs) and solar cells, but the growth of aligned organic NW arrays of a general material is challenging. ${ }^{19-22}$ Although approaches have been demonstrated using an anodic aluminum oxide (AAO) template-based technique, ${ }^{23}$ the alignment of the NWs after removing the template is rather distorted and bunched. ${ }^{24,25}$ A new technique is required to fabricate polymer NW (PNW) arrays with good control over orientation, dimensionality, and location.

Recently, we have demonstrated a novel technique for fabrication of patterned and aligned polymer nanowire arrays on a wafer-level substrate of any material. ${ }^{26}$ By creating a designed pattern on a spin-coated polymer film using techniques such as stamping or microtip writing, plasma etching results in the formation of aligned PNW arrays distributed according to the pattern. It is suggested that the formation mechanism of the NWs is due to a dependence of the size of the ion sample interaction volume on the local incident angle of the ion at the modulated surface. This establishes a new technique for largescale fabrication of organic NW arrays.

In this paper, we expand the technique for fabricating aligned NWs of any polymer with a control over the growth density and length to meet the needs of future device fabrications.

\footnotetext{
* Corresponding author. E-mail: zhong.wang@mse.gatech.edu.

Georgia Institute of Technology.

* Peking University.
}

Instead of using surface roughness first created on the polymer film surface, we deliberately sputtered a thin layer of metal nanoparticles on the substrate, which serve as the "nanomasks" for ion irradiation, resulting in the initial roughness for further etching. Polymer NWs to be presented include but are not limited to PMMA (poly(methyl methacrylate)), PS (polystyrene), PDMS (polydimethylsiloxane), PEDOT/PSS (poly(3,4-ethylenedioxythiophene) poly(styrenesulfonate)), PEN (polyethylene naphthalate), PET (polyethylene terephthalate), and Kapton film (Dupont). The mechanism for achieving the density and length control will be discussed.

Our approach for producing the aligned NWs is by a onestep plasma reactive ion etching process (inductively coupled plasma; ICP), which has been introduced previously. ${ }^{26}$ Briefly, a thin polymer layer of interest is spin-coated on a substrate of choice. Before being etched by the ICP, polymer films were coated with different thicknesses of Au using a DC sputter. Ar, $\mathrm{O}_{2}$, and $\mathrm{CF}_{4}$ gases were induced in the ICP chamber with flow ratios of 15.0,10.0, and $30.0 \mathrm{sccm}$ (standard cubic centimeter per minute), respectively. The operation temperature was 55.0 ${ }^{\circ} \mathrm{C}$ with a pressure of $15 \mathrm{mTorr}$. One power source of $400 \mathrm{~W}$ was used to generate a large density of plasma while another power source of $100 \mathrm{~W}$ was used to accelerate plasma ions toward the polymer surface.

Our previous study indicates that the NWs are created by creating surface roughness that results in a local curvature and incidence angle-dependent etching rate, and on the basis of FTIR data, ${ }^{26}$ such an etching process does not change the bonding characteristic of the polymer. In the current study, the surface roughness for a flat polymer film is created by sputtering a thin layer of gold particles, the shadowing effect of which is the main process for creating the surface roughness required for the formation of PNW arrays. The density and length of the NWs can be controlled via controlling the thickness of the sputtered Au particle film and the plasma etching time. A thin film of $\mathrm{Au}$ was sputtered on the polymer surface before the etching process, then we used ICP ionic milling to etch the polymer films, using $\mathrm{Ar}, \mathrm{O}_{2}$, and $\mathrm{CF}_{4}$ as the etching gases. Other metals, such as Pt, Ti, and $\mathrm{Al}$, can also be used as coating materials to get results similar to $\mathrm{Au}$, because they just serve 


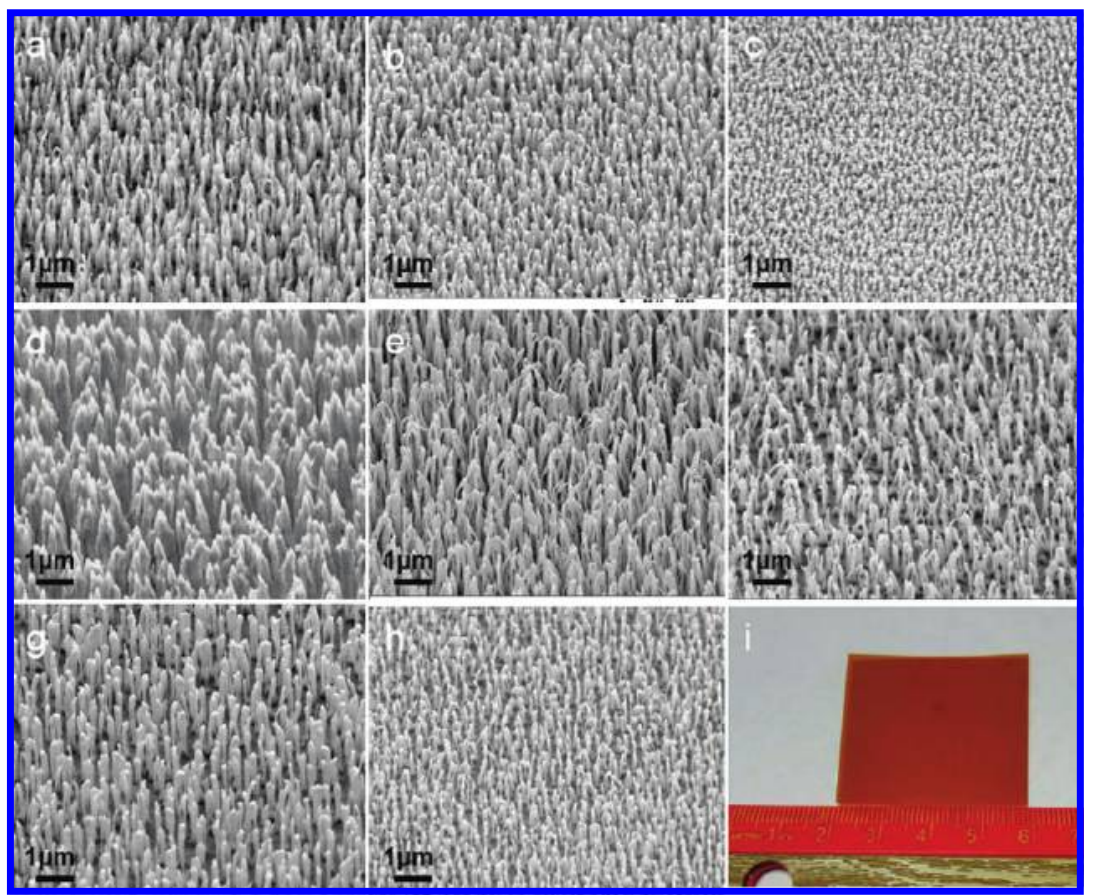

Figure 1. SEM images of fabricated polymer nanowire arrays: (a) PMMA, (b) PS, (c) PDMS, (d) PEDOT/PSS, (e) PEN, (f) PET, (g) Durafilm, and (h) Kapton film. (i) Optical image of $4 \mathrm{~cm} \times 4 \mathrm{~cm}$ Kapton film covered with uniform PNW array.

as a nanoscale mask for creating the surface roughness at the beginning. $\mathrm{Al}_{2} \mathrm{O}_{3}$ deposited by using $\mathrm{ALD}$ (atomic layer deposition) was also used as a coating material to get the PNW array structure.

Figure $1 \mathrm{a}-\mathrm{g}$ shows the scanning electron microscopy (SEM) images of the nanowires fabricated for different polymers, including PMMA, PS, PDMS, PEDOT/PSS, PEN, PET, Durafilm, and Kapton film. Our previous study shows that the etching process does not change the bonding characteristic of the polymer based on FTIR data. ${ }^{26}$ The distribution of the PNWs is rather uniform, and all of them are perpendicular to the substrate surfaces. PMMA solution (in anisole) was spin-coated on $\mathrm{Si}$ wafer, then baked at $180{ }^{\circ} \mathrm{C}$ for $90 \mathrm{~s}$. Before the ICP etching, a 5-nm-thick $\mathrm{Au}$ film was sputtered onto the PMMA surface. Ar, $\mathrm{O}_{2}$, and $\mathrm{CF}_{4}$ were induced into the ICP chamber while the pressure of the chamber was pumped to 15.0 mTorr. The operation temperature was $55.0^{\circ} \mathrm{C}$. After 2 min of etching, the structure shown in Figure 1a was received. This fabrication process is readily scalable by using a large-size substrate and a larger plasma chamber. Figure 1i shows a substrate of $4 \mathrm{~cm} \times$ $4 \mathrm{~cm}$ that is covered uniformly by a PNW of Kapton.

Density control of the PNW arrays can be easily achieved via controlling the thickness of the deposited $\mathrm{Au}$ in this method. Figure 2 shows the density control of different kinds of polymer films. Two minutes of the standard process was used for all of the polymer nanowire arrays. Different thicknesses of Au can make the density of the polymer nanowire array change from $10^{4} \mathrm{~mm}^{-2}$ to $10^{7} \mathrm{~mm}^{-2}$ when the thickness of the Au film is less than $5 \mathrm{~nm}$. After the thickness of the Au film increases to $>5 \mathrm{~nm}$, the density remains the same at $\sim 10^{7} \mathrm{~mm}^{-2}$, even for different types of polymers. Figure 3 shows the SEM images of a PNW array on Kapton films with different densities. The densities of PNW arrays increased when the thicknesses of the $\mathrm{Au}$ increased at the beginning. After the thickness of Au reached a thickness of 1-4 nm (varieties of different polymers), the densities of the PNW arrays stayed at the same order.

The length of the PNWs can be easily controlled via using different processing times. Figure 4 shows the length control

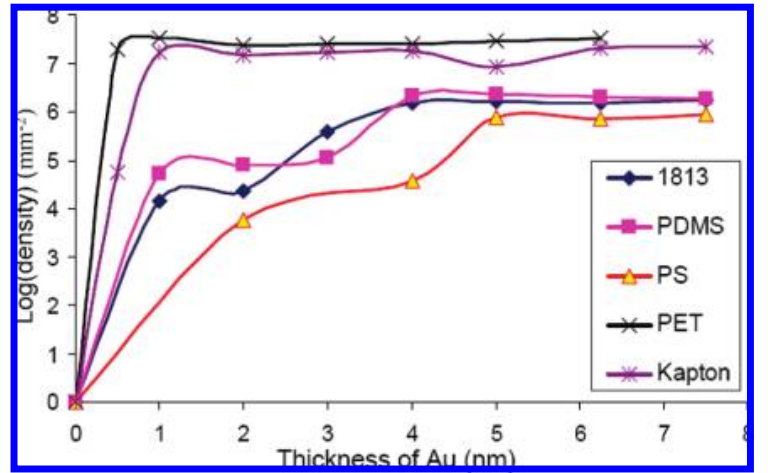

Figure 2. Density-controlled fabrication of PNWs of photoresist 1813 , PDMS, PS, PET, and Kapton film by sputtering different thicknesses of Au particles on the surfaces.

of the nanowires. The length shows a linear increase as the processing time increases. The general etching ratio is about $300-400 \mathrm{~nm} / \mathrm{min}$, whereas the diameter of the nanowire remains as a constant, around $100 \mathrm{~nm}$. After $30 \mathrm{~min}$ of processing, the length of the polymer nanowire is more than $10 \mu \mathrm{m}$ in length, and the aspect ratio can reach up to more than 700 (Figure 4b). Because of the use of ICP, plasma ions were accelerated through a potential difference to make the etching selectivity much better than other plasma etching methods, such as reactive ion etching.

Figure 5 shows the SEM images of PMMA PNWs arrays created using a TEM copper grid. We covered the PMMA film with a TEM copper grid before sputtering gold so that some part of the film is covered with Au, while others are not. Then we removed the copper grid and etched the PMMA film with ICP. After the ICP etching, we received the patterned PNW array. In the SEM images, we can see clearly that PNW arrays were created at the areas that were covered initially with gold, and there were no PNWs at the surfaces that were not covered by gold particles. It indicates that gold nanoparticles play a key role in the formation of PNWs. When the sputter time was very short, the Au coverage was rather low and the nanoparticles were very small so that the particles were easily knocked off 

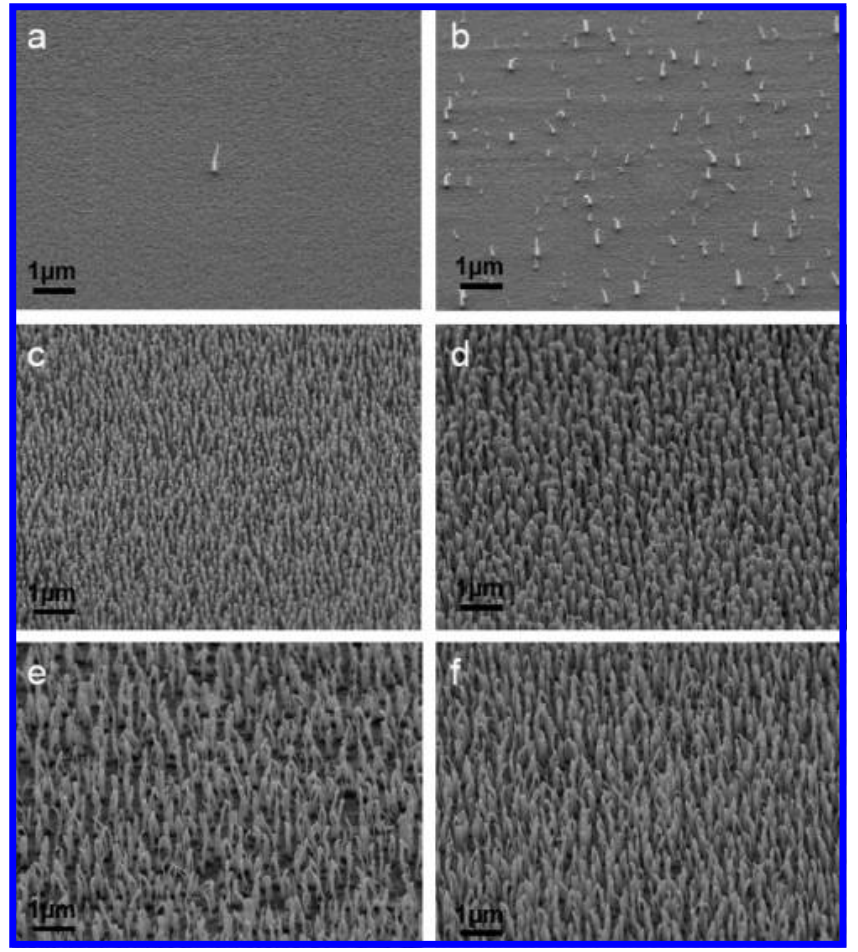

Figure 3. SEM images of density-controlled fabrication of PNW arrays of Kapton by covering the initial surface with (a) 0.75 , (b) 1.5 , (c) 3 , (d) 4.5 , (e) 10 , and (f) $15 \mathrm{~nm}$ of Au before ICP etching.

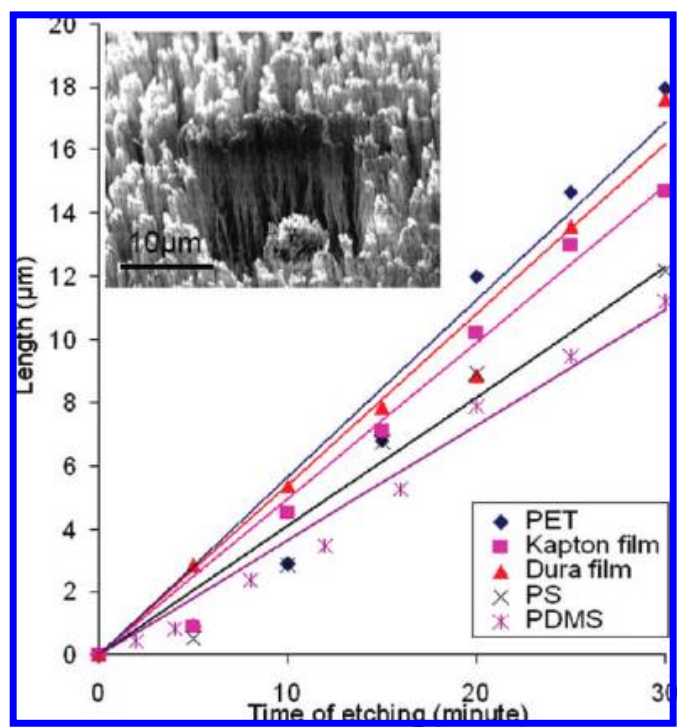

Figure 4. Length-controlled growth of NWs of PET, Kapton film, Durafilm, PS, and PDMS. The inset is a SEM image of a PNW array on Durafilm after 30 min of etching.

by the plasma from the substrate and they could not be effective to serve as the nanomasks for ion etching, resulting in rather sparsely distributed NWs. With an increase in sputtering time, larger and thicker gold particles are formed, which can effectively serve as nanomasks for creating the required surface roughness during initial ion etching for forming the NWs. Accordingly, the density of the NWs also increases. After the $\mathrm{Au}$ covered the entire polymer surface, the density remained at a steady value.

To understand the formation process of the PNW arrays, we use energy-dispersive X-ray spectroscopy (EDS) to analyze the element composition of the polymer surface after the ICP etching. Figure 6a shows the atomic force microscopy (AFM)

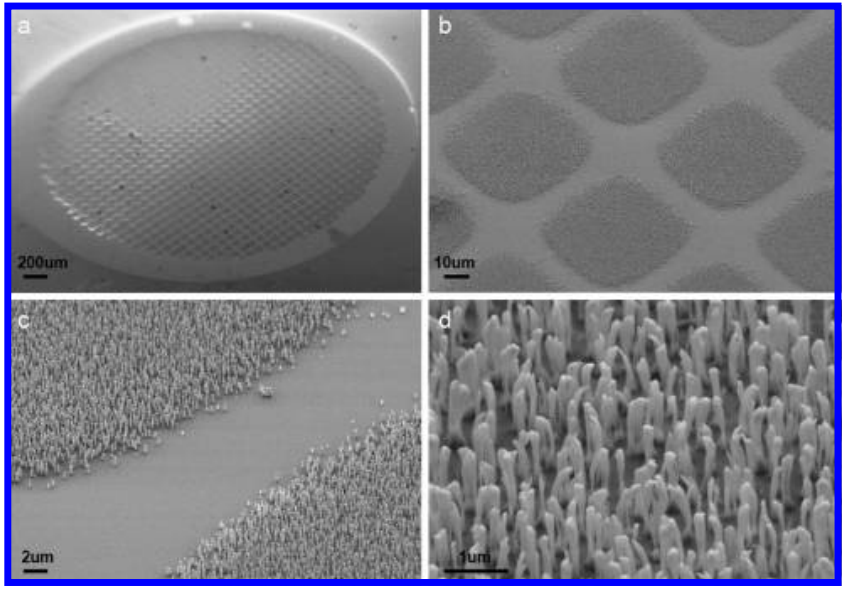

Figure 5. SEM images of PNW arrays of PMMA created using a TEM grid as a mask from low to high magnification.

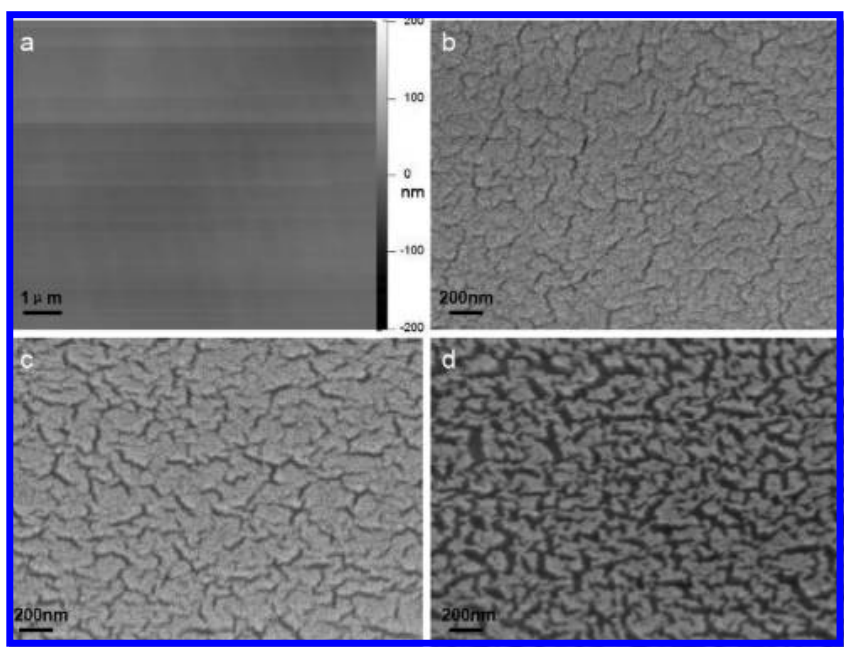

Figure 6. (a) AFM image of Au-coated Kapton film before ICP etching, showing undetectable surface roughness change. Note that the lines in the horizontal direction are due to the tip scanning. (b, c, d) SEM images of Kapton films after ICP etching for (b) 5, (c) 10, and (d) $15 \mathrm{~s}$.

TABLE 1: EDS Analysis of Au Content in the Sample As a Function of Etching Time

\begin{tabular}{cccccc}
\hline time of etching $(\mathrm{s})$ & 0 & 5 & 10 & 15 & 20 \\
\hline atomic percentage of $\mathrm{Au}(\%)$ & 0.28 & 0.25 & 0.22 & 0.08 & 0
\end{tabular}

image of the Kapton film coated with $5 \mathrm{~nm}$ of Au before ICP etching. The morphology of the film did not change after $\mathrm{Au}$ coating, and the roughness was less than $5 \%$. Figure $6 \mathrm{~b}, \mathrm{c}, \mathrm{d}$ shows the SEM images of the Kapton film after ICP etching for 5,10 , and $15 \mathrm{~s}$. The EDS data (Table 1) shows that the atomic percentage of gold decreased with the increase in the etching time, and after $20 \mathrm{~s}$, the gold disappeared on the EDS data. This phenomenon shows that the Au that was sputtered onto the polymer surface just existed on the polymer surface at the very beginning of the ICP etching, and after about $30 \mathrm{~s}$, the gold was sputtered away from the polymer surface. According to Figure 6 and the EDS data, the gold used here just made the polymer surface form a rough morphology. So after the very beginning of the etching process, the mechanism of the nanowire formation is the same as reported previously. ${ }^{26} \mathrm{With}$ the increase in the etching time, the Au nanoparticles located at the tips of the NWs may be knocked off by the ion beam; thus, the final NWs have no $\mathrm{Au}$ at their tops. 
In summary, we developed a one-step method to fabricate large-scale PNW arrays of any organic materials, of which the density and length can be easily controlled via depositing different thicknesses of $\mathrm{Au}$ and using different ICP etching times. This technique uses the masking effect of the Au particles deposited on the surface. Our study demonstrates an effective approach for creating functional organic NW arrays for applications in sensors, electronics, biomaterials and energy materials.

Acknowledgment. This research was supported by DARPA (Army/AMCOM/REDSTONE AR, W31P4Q-08-1-0009), BES DOE (DE-FG02-07ER46394), and NSF (DMS 0706436, CMMI 0403671). Hao Fang thanks the fellowship support by the China Scholarship Council (CSC) (No. 20073020). Thanks to Yue Shen and $\mathrm{Su}$ Zhang for stimulating discussions.

\section{References and Notes} 1289

(1) Cui, Y.; Wei, Q. Q.; Park, H. K.; Lieber, C. M. Science 2001, 293,

(2) Patolsky, F.; Timko, B. P.; Yu, G.; Fang, Y.; Greytak, A. B.; Zheng,

G.; Lieber, C. M. Science 2006, 313, 1100

(3) Pauzauskie, P. J.; Yang, P. Mater. Todav 2006, 9, 36

(4) Zhong, Z. H.; Wang, D. L.; Cui, Y.; Bockrath, M. W.; Lieber, C. M. Science 2003, 302, 1377.

(5) Qin, Y.; Wang, X. D.; Wang, Z. L. Nature 2008, 451, 809.

(6) Wang, X. D.; Song, J. H.; Liu, J.; Wang, Z. L. Science 2007, 316, 102.

(7) Persson, A. I.; Larsson, M. W.; Stenström, S.; Ohlsson, B. J.; Samuelson, L.; Wallenberg, L. R. Nat. Mater. 2004, 3, 677681.

(8) Adhikari, H.; Marshall, A. F.; Chidsey, C. E. D.; McIntyre, P. C. Nano Lett. 2006, 6, 318
(9) Bjork, M. T.; Thelander, C.; Hansen, A. E.; Jensen, L. E.; Larsson, M. W.; Wallenberg, L. R.; Samuelson, L. Nano Lett. 2004, 6, 1621.

(10) Cole, J. J.; Wang, X.; Knuesel, R. J.; Jacobs, H. O. Nano Lett. 2008, 8, 1477.

(11) Wagner, R. S.; Ellis, W. C. Appl. Phvs. Lett. 1964, 4, 89.

(12) Kodambaka, S.; Tersoff, J.; Reuter, M. C.; Ross, F. M. ; Science 2007, 316, 729 .

(13) Kirkham, M.; Wang, Z. L.; Snyder, R. L. Nanotechnology 2008, $19,445708$.

(14) Barbe, C. J.; Arendse, F.; Comte, P.; Jirousek, M.; Lenzmann, F.; Shklover, V.; Gratzel, M. J. Am. Chem. Soc. 1997, 80, 3157.

(15) Yu, S. H.; Qian, Y. T.; Shu, L.; Xie, Y.; Yang, L.; Wang, C. S. Mater. Lett. 1998, 35, 116.

(16) Xu, S.; Wei, Y.; Kirkham, M.; Liu, J.; Mai, W.; Davidovic, D.; Snyder, R. L.; Wang, Z. L. J. Am. Chem. Soc. 2008, 130, 14958.

(17) Kuykendall, T.; Pauzauskie, P. J.; Zhang, Y. F.; Goldberger, J.; Sirbuly, D.; Denlinger, J.; Yang, P. D. Nat. Mater. 2004, 3, 524.

(18) Xu, D. S.; Xu, Y. J.; Chen, D. P.; Guo, G. L.; Gui, L. L.; Tang, Y. Q. Chem. Phvs. Lett. 2000, 325, 340.

(19) Wanekaya, A. K.; Chen, W.; Myung, N. V.; Mulchandani, A. Electroanalvsis 2006, 18, 533.

(20) Thurn-Albrecht, T.; Schotter, J.; Kästle, G. A.; Emley, N.; Shibauchi, T.; Krusin-Elbaum, L.; Guarini, K.; Black, C. T.; Tuominen, M. T.; Russell, T. P. Science 2000, 290, 2126.

(21) Chen, Y.; Luo, Y. Adv. Mater. 2009, 21, 2040.

(22) Cheng, K. H.; Zhong, Y.; Xie, B. Y.; Dong, Y. Q.; Hong, Y.; Sun, J. Z.; Tang, B. Z.; Wong, K. S. J. Phvs. Chem. C 2008, 112, 17507.

(23) Liu, J.; Lin, Y.; Liang, L.; Voigt, J. A.; Huber, D. L.; Tian, Z. R.; Coker, E.; Mckenzie, B.; Mcdermott, M. J. Chem.-Eur. J. 2003, 9, 604.

(24) Lu, M.; Li, X. H.; Li, H. L. Mater. Sci. Eng. 2002, A334, 291.

(25) Xiong, S. X.; Wang, Q.; Xia, H. S. Mater. Res. Bull. 2004, 39, 1569.

(26) Morber, J. R.; Wang, X.; Liu, J.; Snyder, R. L.; Wang, Z. L. $\underline{A d v}$. Mater. 2009, 21, 2072.

JP907072Z 